\title{
La cadena de suministro del mezcal del estado de Zacatecas Situación actual y perspectivas de desarrollo
}

Gabriel López Nava

Universidad Popular Autónoma del estado de Puebla

glopez@bits-group.com

\section{José Luis Martínez}

Flores

Universidad Popular Autónoma del estado de Puebla joseluis.martinez01@upaep.mx

Judith Cavazos Arroyo Universidad Popular Autónoma del estado de Puebla judith.cavazos@upaep.mx

\section{Yésica Mayett Moreno}

Universidad Popular Autónoma del estado de Puebla yesica.mayett@upaep.mx

\section{Resumen}

El mezcal es una bebida alcohólica hecha de la planta de agave que se cultiva en Zacatecas, México, uno de los siete estados que posee la denominación de origen (DO) y el segundo productor de agave y mezcal en el país; de ahí que la industria del agave-mezcal sea una actividad estratégica del estado. Dado que varios agentes interactúan en forma desarticulada en la industria, los principales objetivos de este estudio son: 1) obtener información del proceso global, y 2) desarrollar un modelo táctico de planeación de la cadena de suministro de esta industria. A partir de una investigación preliminar se identifica la información básica de los procesos de negocio obtenida por medio de encuestas y entrevistas, lo que permitió el diseño de un modelo táctico de planeación de la cadena de suministro. Este análisis considera las relaciones entre empresas pequeñas y medianas (Pyme). Los resultados permitieron la obtención de información relacionada con la industria; la identificación de las áreas de oportunidad de los agentes de la cadena; y la evaluación del potencial y restricciones de la cadena de suministro del agave-mezcal del estado de Zacatecas.

Palabras clave: mezcal, Zacatecas, Pyme, cadena de suministro. 


\title{
Mezcal Supply Chain in Zacatecas State. Current situation and perspectives
}

\begin{abstract}
Mezcal is a distilled alcoholic beverage made from the agave plant native from Zacatecas, Mexico, one of the seven States in Mexico which owns mezcal designation of origin (DO) and it is the second agave producer in the country; therefore; the agave-mezcal industry is a strategic activity in the State. Since several agents interact in a non-articulated way in the industry, the main objectives of this study are: 1) to get reliable information of the whole process, and 2) to develop a supply chain tactical planning model of this industry. Initially, an preliminary research identifies basic information related to business processes, using structured primary data: interviews and surveys. Taking into account the gathered basic information, the tactical planning model of the supply chain is developed. This analysis considers the relationships among small-to medium sized enterprises (SMEs). The results of this study allowed obtaining information related to the industry; the identification of specific opportunity areas in the agents' interaction, and the evaluation of the potential and constraints that the Zacatecas' agave-mezcal supply chain has.
\end{abstract}

Keywords: mezcal, Zacatecas, small and medium enterprises, supply chain.

\section{Introducción}

El estudio de la cadena de suministro del mezcal del estado de Zacatecas abarca desde la plantación y cosecha del agave hasta la obtención del producto final que es el mezcal mismo y su comercialización.

En México se producen anualmente 365 mil toneladas de agave. Dicha producción corresponde a los siete estados con denominación de origen del mezcal, los cuales son: Durango, Guanajuato, Guerrero, Oaxaca, San Luis Potosí, Tamaulipas y Zacatecas (Instituto Mexicano de la Propiedad Intelectual, 1994).

Las condiciones socioeconómicas de los productores agrícolas y algunos fabricantes de mezcal incorporados a la industria son de alta y muy alta marginalidad. Algunos de los fabricantes de mezcal aún carecen de tecnología moderna, por lo que obtienen su producto de manera artesanal mediante procesos poco eficientes. Otros han modernizado su proceso incorporándose al campo agroindustrial (Presupuesto de Egresos de la Federación para el Ejercicio Fiscal, 2009 y 2010).

En el nivel nacional, la industria del mezcal cuenta con la infraestructura descrita en el cuadro 1. 


\section{Cuadro 1 \\ Infraestructura de la Industria del Mezcal en México}

\begin{tabular}{c|c}
\hline Concepto & Dato \\
\hline Productores agrícolas (miles) & 30 \\
\hline Plantaciones de agave (miles hectáreas) & 50 \\
\hline Poblaciones naturales (miles hectáreas) & 170 \\
\hline Cantidad de fábricas & 625 \\
\hline Cantidad de envasadoras & 80 \\
\hline Cantidad de marcas de mezcal & 130 \\
\hline Producción de mezcal (millones de litros) & 8 \\
\hline Cantidad de empleos & 5270 \\
\hline Ingresos (millones de pesos) & 1700 \\
\hline
\end{tabular}

Fuente: Presupuesto de Egresos de la Federación para el Ejercicio

Fiscal, 2010

Para certificar la calidad del mezcal, en octubre de 2004 se creó el Consejo Mexicano Regulador de la Calidad del Mezcal (COMERCAM), organismo de certificación acreditado por la Dirección General de Normas de la Secretaría de Economía para evaluar la conformidad de la NOM-070-1994-SCFI-Bebidas-Alcohólicas-Mezcal-Especificaciones; esta norma exige el certificado vigente en el envasado y etiquetado para la comercialización; además, incluye un censo de los predios plantados de agave y un esquema de prueba de laboratorio, así como la vigilancia en todo el proceso de producción. Sin dichas normas el producto no podría comercializarse.

Aunque la cadena productiva del agave-mezcal se ha visto apoyada con $500 \mathrm{mi}$ llones de pesos del presupuesto federal (de 2004 a 2009) -principalmente para el estudio de plantaciones, equipamiento de fábricas y desarrollo de programas promocionales (Presupuesto de Egresos de la Federación para el Ejercicio Fiscal, 2009 y 2010)-; aún existen serias deficiencias en la integración de las diferentes etapas del proceso productivo.

Por su parte, Zacatecas es uno de los siete estados de la República Mexicana que posee la denominación de origen del mezcal (Dirección General de Normas de la Secretaría de Economía, 1994) y es el segundo en producción de agave en el país (COMERCAM, 2011). Esto reconoce que el sistema producto agave-mezcal es una actividad estratégica para la entidad y, por tanto, requiere contar con un estudio sobre su situación actual y perspectivas de desarrollo. 


\section{Antecedentes}

En la actualidad, la producción y el consumo de mezcal están recobrando espacios importantes en la cultura de México y en algunos lugares del mundo. Se le reconoce como la bebida más mexicana, y en la categoría de bebidas espirituosas es una de las más puras (Consejo Mexicano de Productores de Maguey Mezcal, 2009).

La palabra mezcal tiene su origen en vocablos de la lengua náhuatl metl que significa maguey e ixcalli que significa cocer. Estos dos términos dan lugar a la palabra mexcalli, cuya traducción literal es "maguey cocido". De acuerdo con la Norma Oficial Mexicana (1994), el mezcal es una "bebida alcohólica regional obtenida por destilación y rectificación de mostos preparados directa y originalmente con los azúcares extraídos de las cabezas maduras de los agaves [...] previamente hidrolizadas o cocidas, y sometidas a fermentación alcohólica con levaduras, cultivadas o no, siendo susceptible de ser enriquecido." "El mezcal es un líquido de olor y sabor sui generis de acuerdo con su tipo. Es incoloro o ligeramente amarillento cuando es reposado o añejado en recipientes de madera de roble blanco o encino, o cuando se aboque sin reposarlo o añejarlo".

La planta de agave, que es nativa de México, tiene hojas en roseta con características de rigidez, opacidad, simetría radial, almacenamiento de agua y captación de radiación fotosintética activa. El género agave es uno de los ocho que forman la extensa familia botánica Agavacea. Existen unas 200 variedades de agave, entre las más conocidas están las que se muestran en la figura 1.

\section{Figura 1}

Ejemplos de agave

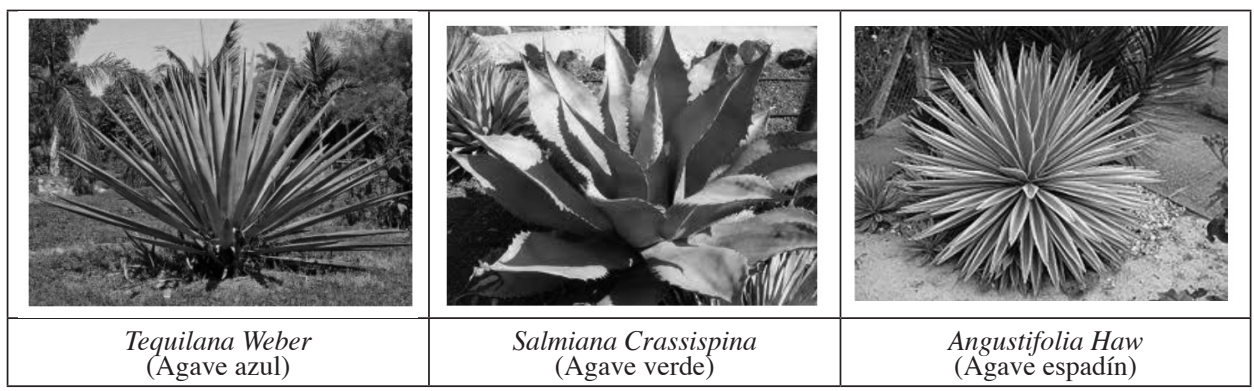

Fuente: Imágenes públicas de internet (2012) 
¿Es tequila o es mezcal? Debido a la amplia difusión y comercialización que ha tenido el tequila, tanto en el nivel nacional como mundial, es menester establecer la diferencia que existe entre ambas bebidas. El tequila es producido únicamente a partir de la planta de agave Tequilana Weber (agave azul) y debe contener desde un $51 \%$ hasta un $100 \%$ de agave. En tanto que el mezcal se produce a partir de diversos tipos de agave, incluido el Tequilana Weber y debe ser producido con un $100 \%$ de agave (Norma Oficial Mexicana, 1994, 2005).

A partir de la NOM-070-SCFI-1994-Bebidas-Alcohólicas-Mezcal y la NOM-006-SCFI-2005-Bebidas-Alcohólicas-Tequila queda establecido que las especificaciones para la certificación del mezcal son más restrictivas que las del tequila, particularmente en lo que se refiere a los alcoholes superiores (ver cuadro 2).

\section{Cuadro 2}

Comparativo de especificaciones de grado alcohólico

\begin{tabular}{l|c|c|c|c}
\hline & \multicolumn{2}{|c|}{$\begin{array}{c}\text { Mezcal } \\
\text { NOM-070 }\end{array}$} & \multicolumn{2}{c}{$\begin{array}{c}\text { Tequila } \\
\text { NOM-006 }\end{array}$} \\
\hline $\begin{array}{l}\text { Especificaciones de } \\
\text { Grado Alcohólico }\end{array}$ & $\begin{array}{c}\text { Nivel } \\
\text { mínimo }\end{array}$ & Nivel máximo & $\begin{array}{c}\text { Nivel } \\
\text { mínimo }\end{array}$ & $\begin{array}{c}\text { Nivel } \\
\text { máximo }\end{array}$ \\
\hline $\begin{array}{l}\text { Porcentaje de alcohol } \\
\text { en volumen a } 20^{\circ} \mathrm{C}\end{array}$ & 36.0 & 55.0 & 35.0 & 55.0 \\
\hline $\begin{array}{l}\text { Alcoholes superiores } \\
\mathrm{mg} / 100 \mathrm{ml}\end{array}$ & 100.0 & 400.0 & 20.0 & 500.0 \\
\hline $\begin{array}{l}\text { Metanol } \\
\mathrm{mg} / 100 \mathrm{ml}\end{array}$ & 100.0 & 300.0 & 30.0 & 300.0 \\
\hline
\end{tabular}

Fuente: Norma Oficial Mexicana $(1994,2005)$

Como consecuencia de lo anterior, se puede decir que el tequila es un tipo de mezcal y su nombre apropiado debería ser: "Mezcal de tequila"; de hecho, el nombre original del tequila era: "Vino mezcal de tequila".

Una característica particular y muy valiosa que tiene la industria del mezcal es que tiene exclusividad de producción, llamada denominación de origen (DO). Una DO es un signo distintivo que se refiere a un nombre geográfico que se destina a un producto, el cual está asociado a las condiciones especiales (factores humanos, naturales, entre otros de la misma región) que le dan un carácter único. Al ser la denominación de origen un signo distintivo referente a una región geográfica, este producto no puede ser apropiado en forma individual o privada, lo que significa que es un elemento de Patrimonio Nacional. 
El estado de Zacatecas ocupa el segundo lugar en el nivel nacional en la producción de mezcal; en 2005 apenas tenía una capacidad instalada de 1.5 millones de litros anuales; para finales de 2010, se elevó a 4.8 millones y se utilizaba el $40 \%$ de dicha capacidad, lo que representaba una producción anual de dos millones de litros. La venta de exportación representaba el 10\% de la producción anual.

\section{Situación actual}

La industria del mezcal en el estado de Zacatecas está conformada por aproximadamente mil productores de agave y 22 fábricas de mezcal (micro, pequeñas y medianas empresas), las cuales en conjunto fabrican 60 distintos tipos de mezcal, pues cada empresa produce por lo menos dos modalidades diferentes. Zacatecas cuenta predominantemente con dos zonas de producción de agave y mezcal, mismas que se enuncian a continuación.

- Región de los Cañones de Tlaltenango y Juchipila. Esta región se localiza en el sur del estado y se denomina así por su configuración fisiográfica. Estos cañones se encuentran principalmente ubicados en los municipios de Trinidad García de la Cadena, Teúl de González Ortega, Jalpa y Juchipila. De éstos, el municipio de Teúl de González Ortega concentra la mayor producción de mezcal del estado. En esta región se siembra la planta Tequilana Weber o agave azul, de la cual se produce el mezcal de esta zona. La región de los cañones se ve fortalecida por la comercialización del agave debido a su cercanía con el estado de Jalisco.

- Región de Pinos. Esta región se localiza en el sureste del estado en una zona semidesértica, cuyas características propician la producción de maguey y nopal. Esta región comprende los municipios de Pinos y Villa Hidalgo, que se localizan en el altiplano potosino-zacatecano; en él se desarrollan cinco especies de agave: Scabral Potosintesis, Parrasana Berger, Americana variedad Oaxacensis, Mapisaga y Salmiana Crassispina o agave verde; esta última especie es la más abundante. A la fecha, las empresas de esta región trabajan en el aprovechamiento de la planta Salmiana Crassispina, la cual se usa para producir mezcal, mieles endulzantes, alimentos alternos, gusanos de maguey, forrajes para ganado, material para construcción de vivienda rural y artículos para usos ornamentales, entre otros.

En estos momentos el robustecimiento de esta agroindustria se evidencia por el logro de los siguientes resultados: 
- Constitución del Sistema-Producto Maguey-Mezcal (en el nivel nacional)

- Registro de la Denominación de Origen Mezcal (junto con otros seis estados)

- Cumplimiento de la Norma NOM-070-SCFI-1994-Bebidas-AlcohólicasMezcal

- Creación del Consejo Mexicano para la Certificación de la Calidad del Mezcal (COMERCAM)

- Fortalecimiento de la infraestructura de las fábricas de mezcal

- Industrialización del proceso de producción de mezcal para hacerlo más eficiente

- Automatización del proceso de envasado

- Incremento en la producción de producto certificado

- Desarrollo de eventos promocionales en el extranjero

\section{Justificación del estudio}

La integración de micro, pequeñas y medianas empresas (productores agrícolas, sociedades de producción rural, fábricas de mezcal, entre otros) en un marco productivo como lo es el Sistema-Producto Agave-Mezcal ha generado resultados importantes gracias a la conformación de una industria con el consecuente beneficio, tanto individualmente como colectivamente. No obstante, aún no se ha consolidado como una agroindustria productiva desde el punto de vista económico y social. Actualmente, se desconoce el nivel de uso del terreno productivo de las plantaciones de agaves; no se tiene claramente identificada la utilización de la capacidad productiva de las fábricas de elaboración de mezcal; se desconocen los niveles de productividad de la producción primaria (cosecha de agave) y secundaria (fabricación de mezcal); no están claramente identificados los factores que obstaculizan la eficiencia de la cadena de suministro. Por lo tanto, el planteamiento del problema se resume en la siguiente pregunta: ¿en qué condiciones de funcionalidad se encuentra la agroindustria del mezcal en el estado de Zacatecas en los siete años de estudio?

Objetivo general

- Diagnosticar el estado actual del funcionamiento de la cadena agroindustrial (producción-consumo) del mezcal en el estado de Zacatecas; asimimos, desarrollar un modelo táctico de planeación para la cadena de suministro. 
Objetivos particulares

- Realizar un diagnóstico de la percepción de las condiciones que prevalecen en la cadena de suministro de la industria del mezcal, según la opinión de algunos productores de agave, fabricantes de mezcal y líderes de opinión.

- Identificar el perfil de algunos de los actores dentro de esta cadena.

- Identificar los elementos y actores clave para el diseño del modelo táctico de la cadena de suministro de la industria del mezcal.

- Determinar los niveles de utilización de las capacidades instaladas de la producción primaria y secundaria.

- Desarrollar un modelo matemático para la evaluación de la cadena de suministro de la industria del mezcal.

\section{Metodología de investigación}

La primera parte del presente estudio fue evaluar el desempeño de las actividades de las organizaciones implicadas en la cadena de suministro, utilizando el modelo de cadena de valor de Porter (1985); en él se enuncian las actividades primarias cuyo objetivo es crear valor para el cliente final. La gestión estratégica sobre estas actividades contribuye a la identificación de fuentes de ventajas competitivas, por un lado; por otro lado, están las actividades de apoyo que sirven de soporte y contribuyen como habilitadores de la empresa.

Por lo que respecta a la región de estudio, ésta comprendió únicamente los municipios localizados al sur del estado donde es cultivado el agave Tequilana Weber y donde se localizan las fábricas de mezcal.

Nuestra investigación se aplicó en las comunidades y rancherías donde se siembra el agave; en los municipios donde están instaladas las fábricas de mezcal y en las oficinas y centros de operación de los líderes de opinión. La cantidad total de productores de agave fue de 933; mientras que la cantidad total de fábricas de mezcal de 25 y los líderes de la industria de 35 . El tamaño de la muestra consistió en 30 productores de agave, 16 fábricas de mezcal y 13 líderes de opinión.

Para llevar a cabo lo anterior, se diseñaron tres instrumentos para recabar la información: dos encuestas semiestructuradas (preguntas abiertas y cerradas de opción múltiple y de opción determinante, de 65 ítems, algunas con escala Likert [valores 
1 a 7], administradas por el investigador, sin ocultamiento, para productores de agave y fabricantes de mezcal). La tercera fue una entrevista semiestructurada para líderes de opinión.

Para realizar la prueba de consistencia interna del instrumento se utilizó el coeficiente de confiabilidad: Alfa de Cronbach (1951) [Ecuación 1].

$$
\propto=\left[\frac{K}{K-1}\right]\left[1-\frac{\sum_{i}^{K} V_{i}}{V_{t}}\right]
$$

Para el caso particular, el número de reactivos aplicados fue de 33, de donde se obtuvo un Alfa de 0.8453 . Debido a que se obtuvo un valor superior a 0.8 , se pudo concluir que la métrica de la encuesta era confiable.

En el caso de los productores de agave, con el fin de obtener un menor número de encuestas para ser realizadas se llevó a cabo un muestreo aleatorio estratificado por municipio con un nivel de confianza del $95 \% ; \mathrm{p}=\mathrm{q}=0.5$; por medio del cual se obtuvo una muestra de 130 .

\section{Análisis de la información}

Se empleó el análisis univariado (Ostle, 1965) para productores de agave y fabricantes de mezcal, así como el cálculo de sumas, promedios, desviaciones estándar, frecuencias, modas de informaciones básicas, entre otras.

Asimismo, se utilizó el análisis multivariado (Smith, 2002), específicamente el análisis de componentes principales. Esta técnica fue utilizada con el fin de identificar la estructura de las variables iniciales para crear un nuevo conjunto de componentes que reemplazara el conjunto original. Las variables utilizadas fueron las relacionadas con el modelo de Porter en las encuestas realizadas con los productores de agave. La medida de adecuación KMO tuvo un valor suficientemente cercano a la unidad. La prueba de esfericidad de Bartlett fue menor a 0.001. Estos dos valores probaron la validez del método empleado (Pérez, 2005).

El análisis cualitativo (David, 2008) se efectuó a partir de la información proporcionada por los líderes de opinión, los cuales son tanto actores clave de la cadena, como agentes externos que conocen bien el desempeño de la misma; por ello, se utilizaron las respuestas a las preguntas abiertas en el cuestionario. A partir del 
análisis cualitativo se determinaron fortalezas, debilidades, oportunidades y amenazas (FODA).

La segunda parte del presente estudio consistió en la formulación de un modelo matemático con la intención de evaluar una forma de optimizar el desempeño de la cadena de suministro desde un punto de vista táctico. Para tal efecto, los resultados del estudio preliminar contribuyeron significativamente para contar con información y poder realizar la planeación del proceso productivo de la agroindustria.

Para el desarrollo del modelo se utilizó el método de planeación de la demanda agregada (Vollmann et al., 1992), el cual sirve para aplicarse en el nivel táctico y tiene como objetivo fundamental establecer los niveles de producción en unidades agregadas a lo largo de un horizonte determinado, de tal forma que se satisfagan las necesidades existentes. Este método busca mantener los niveles mínimos de costos y un buen nivel de servicio al cliente.

El término agregado, en este nivel de planeación, implica que las cantidades por producir se deben establecer de manera global o para una medida general de producción; por lo tanto, en la modelación se incorporó la producción de las diferentes fábricas que la componen sin hacer distinción entre sus diversos productos; esto permitió un manejo fácil y comprensible de las unidades de producción dentro del plan.

Se eligió este método debido a que la planeación de la demanda agregada establece una relación entre las decisiones sobre el empleo de insumos, uso de las instalaciones, los niveles de producción, políticas de inventario a mantener y la programación en un horizonte de tiempo. En esencia, el conocimiento de estas variables permitirá determinar el nivel de producción requerido para cumplir con las expectativas de la demanda tomando en consideración las restricciones de recursos disponibles, minimizando los costos totales.

Este modelo de nivel táctico realiza una planeación de mediano plazo sobre los recursos productivos disponibles: cultivos de agave y fábricas de mezcal; además, tiene una importante repercusión económica, pues a este nivel se deciden los planes de aprovisionamiento, la política de almacenamiento y el uso de la capacidad instalada. 
Los supuestos del modelo fueron: 1) el horizonte de tiempo empleado es de siete años, el cual corresponde con los años de maduración del agave; 2) para efectos de simplicidad, se manejó una correspondencia entre las unidades de producción y las de venta; la unidad de medida será un litro para ambos conceptos; 3) en el modelo desarrollado se emplearon pesos constantes, de modo que no existe efecto por inflación o cambios de precio en el tiempo; 4) con base en el estudio preliminar sobre las plantaciones de agave existentes en el Estado se determinó el volumen de materia prima útil; para efectos de la modelación, se consideró que sólo el $40 \%$ del volumen total por año podría estar disponible dada la falta de cuidado e interés por parte de los productores; 5) se asumió que no existiría resiembra de agave a lo largo del horizonte de tiempo de planeación del modelo; y 6) en el cálculo de la venta y los costos no se incluyeron impuestos de ningún tipo.

Para obtener la solución al planteamiento de planeación de la demanda agregada, se formuló un modelo de programación lineal (ecuaciones 2-5).

$$
\begin{gathered}
\operatorname{Min} C O T=\sum_{i}\left(C P P * U k_{i}+C P S * U l_{i}+C C O * U v_{i}+C A D+C P S * U s_{i}+C I N * U i_{i}+C F I * U f_{i}\right) \\
\left(U i_{i-1}-U f_{i-1}\right)-\left(U i_{i}-\left(U f_{i}\right)+U l_{i}=D E M_{i}\right. \\
U k_{i} \leq C P A_{i} \quad \forall i \\
U l_{i}+U s_{i}=C P M_{i} \quad \forall i
\end{gathered}
$$

\begin{tabular}{|c|c|c|}
\hline $\operatorname{Pr}$ & Precio unitario del mezcal & UM: pesos/litro \\
\hline$C P P$ & Costo de producción primaria & UM: pesos/kilo \\
\hline Cmp & Costo materia prima (piñas de agave) & UM: pesos/kilo \\
\hline$C c$ & Costo de cosecha (jima) & UM: pesos/kilo \\
\hline$C t$ & Costo de traslado de materia prima & UM: pesos/kilo \\
\hline CPS & Costo de producción secundaria & UM: pesos/litro \\
\hline $\mathrm{Cmo}$ & Costo de mano de obra & UM: pesos/litro \\
\hline$C f$ & Costo de fabricación & UM: pesos/litro \\
\hline $\mathrm{Ce}$ & Costo de envaselempaque & UM: pesos/litro \\
\hline $\mathrm{CCO}$ & Costo de comercialización & UM: pesos/litro \\
\hline$C d$ & Costo de distribución & UM: pesos/litro \\
\hline$C v$ & Costo de venta & UM: pesos/litro \\
\hline $\mathrm{Cm}$ & Costo de mercadotecnia & UM: pesos/litro \\
\hline
\end{tabular}

A continuación se enlistan los diferentes elementos de dicho modelo, empezando por los parámetros: 


$\begin{array}{lll}\text { CAD } & \text { Costo de administración } & \text { UM: pesos/año } \\ \text { Cp } & \text { Costo de personal } & \text { UM: pesos/año } \\ \text { Co } & \text { Costo de la oficina } & \text { UM: pesos/año } \\ \text { CSP } & \text { Costo subutilización planta productiva } & \text { UM: pesos/litro } \\ \text { CIN } & \text { Costo de inventario } & \text { UM: pesos/litro } \\ \text { CFI } & \text { Costo de faltante de inventario } & \text { UM: pesos/litro } \\ \text { DEM } & \text { Demanda } & \text { UM:litros } \\ C P A & \text { Capacidad de producción de agave } & \text { UM: kilos } \\ \text { CPM } & \text { Capacidad de producción de mezcal } & \text { UM:litros }\end{array}$

Cálculo de costos:

$C P P=C m p+C c+C t$
$C P S=C m o+C f+C e$
$C C O=C d+C v+C m$
$C A D=C p+C o$

Variables de decisión:

Uv Unidades vendidas

$U M$ : litros

Uk Unidades de agave producidas

UM: kilos

Ul Unidades de mezcal producidas

$U M$ : litros

Ui Unidades de mezcal en inventario

$U M$ : litros

Uf $\quad$ Unidades de mezcal faltante en inventario

UM: litros

Us Unidades de subutilización de planta

$U M$ : litros

Variables calculadas por el modelo:

$\begin{array}{ll}V & \text { Venta } \\ \text { U } & \text { Utilidad } \\ \text { COT } & \text { Costo total }\end{array}$

$U M:$ pesos

$U M:$ pesos

$V=U v_{*} \operatorname{Pr}$

Los objetivos del modelo fueron: 1) determinar el valor de las variables de decisión: kilos de agave empleado, litros de mezcal producidos, vendidos y mantenidos en inventario por año, tales que minimicen los costos totales de los siete años; 2) determinar la utilidad por año y máxima total; 3 ) determinar el porcentaje de uso 
de la materia prima disponible; 4) determinar el porcentaje de uso de la capacidad instalada en fábricas para elaboración de mezcal; y 5) determinar la estructura de costos de la industria del mezcal en el estado.

Las corridas del modelo se realizaron con la herramienta de optimización Solver del software Excel de Microsoft Office ${ }^{\mathrm{TM}}$, en un computador con procesador AMD Athlon ${ }^{\mathrm{TM}}$ II Dual-Core M300 2.00 GHz.

\section{Resultados}

\section{Productores de agave}

Estado de maduración del agave. Existe una marcada tendencia decreciente en la cantidad de hectáreas plantadas de agave por años de maduración (gráfica 1). Esto puede ser indicativo de falta de motivación de los productores con respecto a la resiembra del agave.

El análisis univariado realizado sobre las 17 actividades primarias (definidas con base en el modelo de cadena de valor de Porter) de productores de agave arrojó el siguiente resultado: no se obtuvieron actividades con calificación promedio aprobatoria; sólo la actividad estado del terreno obtuvo en promedio calificación de regular. Las 16 actividades restantes obtuvieron calificaciones promedio reprobatorias.

A partir del análisis de componentes principales aplicado a las actividades primarias (definidas con base en el modelo de cadena de valor de Porter) de los productores de agave se encontró que cinco valores propios fueron mayores que la unidad; por lo tanto, las 17 actividades primarias pudieron ser reducidas a cinco componentes, los cuales explican el 75.2\% de la variación. Con base en este análisis, las actividades de logística externa, seguidas de las de mercadotecnia y ventas, resultaron ser las más susceptibles de mejora. 


\section{Gráfica 1 \\ Siembra de agave (Ha)}

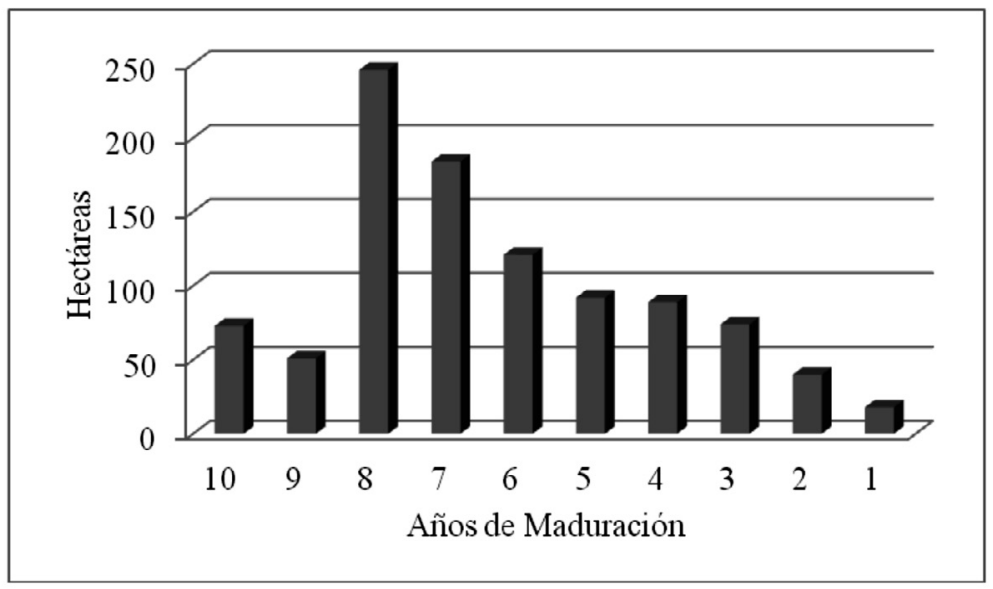

Fuente: López-Nava et al. (2011)

\section{Fabricantes de mezcal}

El porcentaje de utilización de la capacidad de producción de mezcal que se obtuvo fue de sólo el 33\%. Con relación al análisis univariado de las actividades primarias (definidas con base en el modelo de cadena de valor de Porter) de fabricantes de mezcal se obtuvo que del total de 24 actividades, 13 (54.2\%) obtuvieron en promedio calificación aprobatoria; 7 (29.2\%) obtuvieron en promedio calificación regular y $4(16.6 \%)$ obtuvieron en promedio calificación reprobatoria. Las actividades de mercadotecnia y ventas, así como de logística de salida resultaron ser las más susceptibles de mejora.

\section{Líderes de opinión}

Análisis FODA. Las fortalezas más importantes identificadas fueron: producto con denominación de origen, producto autóctono con origen bien definido, productores y mezcaleros con experiencia. Las debilidades más importantes identificadas fueron: bajo precio del agave, falta de proceso de planeación estratégica y cuellos de botella a lo largo de la cadena de suministro. Las oportunidades más importantes identificadas fueron: promoción para obtener provecho de aspectos culturales, místicos y religiosos, producto bien aceptado en el mercado mundial y fuerte demanda de bebidas espirituosas similares en el mercado global. Las amenazas más 
importantes identificadas fueron: percepción generalizada de que el mezcal es de baja calidad, perspectivas de escases de agave en el futuro cercano y que el mezcal es comparado con el tequila.

\section{Perspectivas de desarrollo}

Entre los aspectos críticos que limitan el desarrollo de esta industria están los siguientes: los márgenes de utilidad de sus productos son muy bajos, los insumos (piñas de agave) se deterioran rápidamente una vez que llegaron a su etapa de madurez a los siete años, los precios del agave son sensibles a las fluctuaciones en la demanda, la escasez de producción causa significantes pérdidas de ingresos por ventas no realizadas y por infraestructura no utilizada, así como la administración no apropiada de procesos causa cuellos de botella a lo largo de la cadena productiva, entre otros.

Se calcularon tres escenarios para el pronóstico de la demanda. Para la corrida del modelo se utilizó el escenario más probable. En el cuadro 3 se muestra la producción de agave anual (toneladas) con una tendencia decreciente debido a la actual falta de incentivos para la resiembra.

\section{Cuadro 3}

\section{Capacidad de producción de agave (toneladas)}

\begin{tabular}{c|c|c|c|c|c|c|c}
\hline & \multicolumn{7}{|c|}{ Año } \\
\hline Municipio & $\mathbf{1}$ & $\mathbf{2}$ & $\mathbf{3}$ & $\mathbf{4}$ & $\mathbf{5}$ & $\mathbf{6}$ & $\mathbf{7}$ \\
\hline Apozol & 3408 & 1008 & 2016 & 672 & 336 & 0 & 336 \\
\hline Apulco & 3072 & 2400 & 672 & 2062 & 1008 & 336 & 336 \\
\hline T. García & 4704 & 4704 & 6384 & 4752 & 4416 & 0 & 0 \\
\hline Huanusco & 1008 & 5424 & 1344 & 6864 & 0 & 0 & 0 \\
\hline Jalpa & 5760 & 672 & 8208 & 6864 & 7872 & 7872 & 672 \\
\hline Juchipila & 4080 & 1008 & 1680 & 0 & 0 & 0 & 0 \\
\hline Mezquital & 2736 & 4704 & 2352 & 1008 & 2352 & 0 & 0 \\
\hline Moyahua & 0 & 1344 & 672 & 0 & 672 & 672 & 0 \\
\hline Nochistlán & 10848 & 2688 & 1008 & 672 & 1680 & 0 & 2016 \\
\hline Tabasco & 12624 & 4752 & 1344 & 672 & 1344 & 336 & 0 \\
\hline Tepechitlán & 1008 & 672 & 0 & & 1344 & 0 & 0 \\
\hline Teul & 12816 & 10608 & 3024 & 5424 & 4032 & 1008 & 336 \\
\hline Villanueva & 0 & 1008 & 1344 & 1344 & 0 & 3408 & 2352 \\
\hline Santa María & 0 & 0 & 1008 & 0 & 0 & 0 & 0 \\
\hline TOTAL & $\mathbf{6 2} \mathbf{0 6 5}$ & $\mathbf{4 0 9 9 4}$ & $\mathbf{3 1 0 5 9}$ & $\mathbf{3 0 ~ 3 4 0}$ & $\mathbf{2 5 0 6 1}$ & $\mathbf{1 3 6 3 8}$ & $\mathbf{6 0 5 5}$ \\
\hline
\end{tabular}

Fuente: Elaboración propia con base en información del estudio preliminar 
En el cuadro 4 se muestra la capacidad instalada de producción de mezcal. Dado que la industria se encuentra actualmente con poca actividad, la reactivación de la producción será progresiva. Este hecho fue manejado en el modelo al hacer disponible una capacidad instalada incremental hasta llegar al $80 \%$ del total en el año séptimo.

\section{Cuadro 4}

\section{Capacidad instalada de producción de mezcal}

\begin{tabular}{|c|c|c|c|c|}
\hline No. & Fábrica de mezcal & Localidad & Municipio & $\begin{array}{l}\text { Capacidad de } \\
\text { producción } \\
\text { (miles de } \\
\text { unidades } \\
\text { / año) }\end{array}$ \\
\hline 1 & Hacienda de Tayahua & Tayahua & Villanueva & 18 \\
\hline 2 & Real de Jalpa & Jalpa & Jalpa & 180 \\
\hline 3 & Diamante del Desierto & Juchipila & Juchipila & 450 \\
\hline 4 & El Piñón Gigante de Juchipila & Juchipila & Juchipila & 90 \\
\hline 5 & Empresa Integradora La Caxcania & Moyahua & Moyahua & 432 \\
\hline 6 & Grupo San José de Allende & $\begin{array}{l}\text { Milpillas de } \\
\text { Allende }\end{array}$ & Teul & 90 \\
\hline 7 & Mezcal Hacienda de Bañuelos & Hda de Guadalupe & Teul & 216 \\
\hline 8 & Casa Mezcal Huitzila & Huitzila & Teul & 342 \\
\hline 9 & $\begin{array}{l}\text { Agaveros y Mezcaleros } \\
\text { Zacatecanos }\end{array}$ & Huitzila & Teul & 90 \\
\hline 10 & El Zacatecano & Huitzila & Teul & 342 \\
\hline 11 & El Cambujo & Centro & Teul & 18 \\
\hline 12 & Mezcal El Caxcan & Teul & Teul & 234 \\
\hline 13 & $\begin{array}{l}\text { Mezcales de Calidad Don Aurelio } \\
\text { Lamas }\end{array}$ & Teul & Teul & 360 \\
\hline 14 & Agrícola Curiel & Trinidad García & $\begin{array}{l}\text { Trinidad } \\
\text { García }\end{array}$ & 90 \\
\hline 15 & Empresa Integradora Tres Pueblos & La Ceja & $\begin{array}{l}\text { Trinidad } \\
\text { García }\end{array}$ & 432 \\
\hline 16 & Escorpión La Estanzuela & Trinidad García & $\begin{array}{l}\text { Trinidad } \\
\text { García }\end{array}$ & 90 \\
\hline 17 & Mezcales del Cañón & Trinidad García & $\begin{array}{l}\text { Trinidad } \\
\text { García }\end{array}$ & 90 \\
\hline 18 & Mezcalera Fonseca Villalobos & San Martín & Pinos & 270 \\
\hline \multirow[t]{2}{*}{19} & $\begin{array}{l}\text { Integradora Regional de } \\
\text { Organizaciones }\end{array}$ & San Marín & Pinos & 36 \\
\hline & TOTAL & & & 3870 \\
\hline
\end{tabular}

Fuente: Elaboración propia con base en información del estudio preliminar . 
En el año cero del periodo de la planeación se contaba con un inventario de producto terminado que ascendía a 2.1 millones de litros.

Por otra parte, los valores de los parámetros del modelo se presentan en el cuadro 5.

Cuadro 5

Parámetros del modelo

\begin{tabular}{c|c|c|c|c|c|c}
\hline $\begin{array}{c}\text { Costo de } \\
\text { producción } \\
\text { primaria }\end{array}$ & $\begin{array}{c}\text { Costo de } \\
\text { producción } \\
\text { secundaria }\end{array}$ & $\begin{array}{c}\text { Costo de } \\
\text { comercialización }\end{array}$ & $\begin{array}{c}\text { Costo de } \\
\text { administración }\end{array}$ & $\begin{array}{c}\text { Costo de } \\
\text { subutilización } \\
\text { de la planta } \\
\text { productiva }\end{array}$ & $\begin{array}{c}\text { Costo de } \\
\text { inventario }\end{array}$ & $\begin{array}{c}\text { Costo de } \\
\text { faltante de } \\
\text { inventario }\end{array}$ \\
\hline Pesos/Kilo & Pesos/Kilo & Pesos/Litro & Pesos/Año & Pesos/Litro & Pesos/Litro & Pesos/Litro \\
\hline 1.50 & 2.00 & 8.00 & 3600000.00 & - & - & - \\
\hline 0.20 & 18.00 & 25.00 & 900000.00 & - & - & - \\
\hline 0.14 & 15.00 & 15.00 & - & - & - & - \\
\hline $\mathbf{1 . 8 4}$ & $\mathbf{3 5 . 0 0}$ & $\mathbf{4 8 . 0 0}$ & $\mathbf{4 5 0 0 0 0 0 . 0 0}$ & $\mathbf{1 2 . 0 0}$ & $\mathbf{2 0 . 0 0}$ & $\mathbf{4 0 . 0 0}$ \\
\hline
\end{tabular}

\begin{tabular}{c|c|c|c}
\hline Año & Demanda & $\begin{array}{c}\text { Capacidad } \\
\text { Producción } \\
\text { de Agave }\end{array}$ & $\begin{array}{c}\text { Capacidad } \\
\text { Producción } \\
\text { de Mezcal }\end{array}$ \\
\hline & Litros & Kilos & Litros \\
\hline 1 & 792000 & 62065000 & 774000 \\
\hline 2 & 950400 & 40994000 & 1161000 \\
\hline 3 & 1140480 & 31059000 & 1548000 \\
\hline 4 & 1368576 & 30340000 & 1935000 \\
\hline 5 & 1642292 & 25061000 & 2322000 \\
\hline 6 & 1970751 & 13638000 & 2709000 \\
\hline 7 & 2364902 & 6055000 & 3096000 \\
\hline
\end{tabular}

Información obtenida a partir de la corrida del modelo. Escenario base

La cantidad de mezcal almacenado al inicio del plan (año 0) es de 2.1 millones de litros $y$, puesto que la demanda es baja en los primeros años, esto ocasiona que no se requiera producción de mezcal, consecuentemente, tampoco hay consumo de agave.

Como puede observarse gráfica 2, durante los primeros dos años no se consume agave. A partir del tercer año se empieza a emplear una parte del disponible. Pero es hasta el sexto y séptimo año que se consume el total disponible. A lo largo del horizonte de planeación, el consumo es de sólo el 25\% del total disponible. 
Gráfica 2

Utilización de la capacidad de producción primaria Escenario base

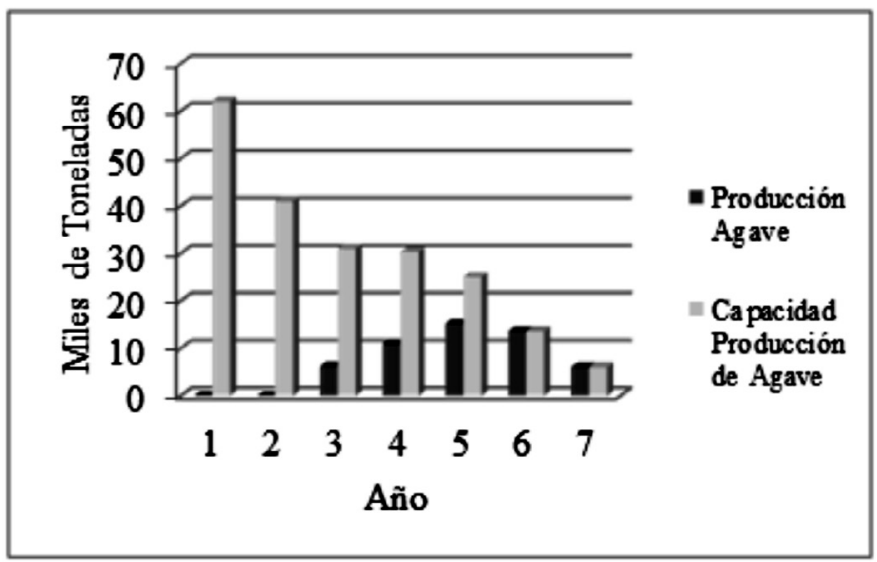

De manera similar al comportamiento del consumo del agave, la capacidad de producción de mezcal de las fábricas es subutilizada (gráfica 3). En los dos primeros años la utilización es nula. Durante los años 3 a 5 se incrementa, pero a partir del sexto se tiene un decremento dramático.

Gráfica 3

Utilización de la capacidad de producción secundaria

Escenario base

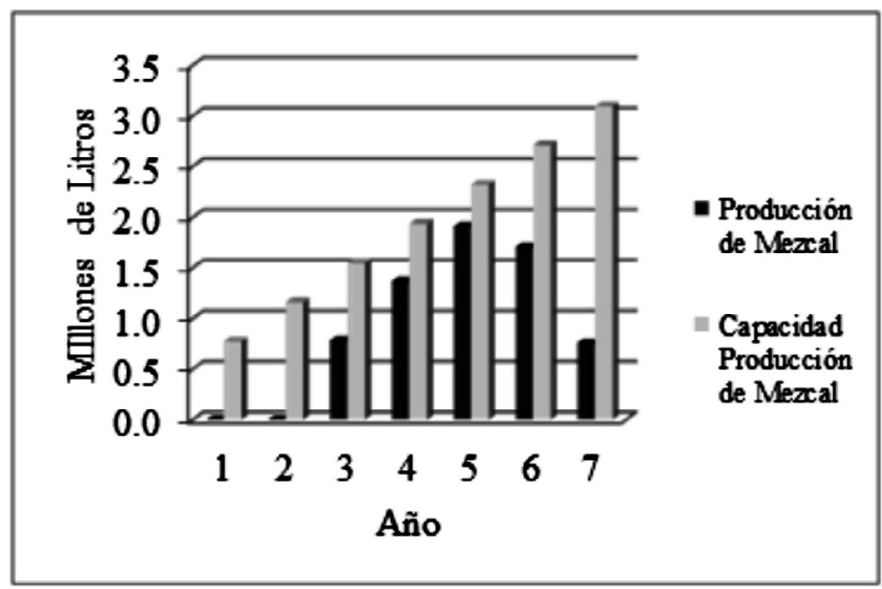


La razón por la cual la producción de mezcal en el séptimo año es tan baja se debe a que en ese año la producción de agave no es suficiente, lo cual también ocasiona que se tenga un faltante de un millón 608 mil 27 litros para cubrir la demanda. A lo largo del horizonte de planeación, la utilización de la capacidad total de las fábricas es de $48 \%$.

En los resultados económicos para los siete años (cuadro 6) la fila de Ajuste corresponde con el decremento por concepto de venta y comercialización no reali$z a d a$, ya que en el modelo se consideró la opción de penalización por faltante de inventario.

\section{Cuadro 6 \\ Estructura de costos, venta y utilidad \\ Escenario base \\ (Millones de pesos)}

\begin{tabular}{|c|c|c|c|c|c|c|c|c|c|}
\hline Año & Venta & $\begin{array}{c}\text { Produc. } \\
\text { primaria }\end{array}$ & $\begin{array}{c}\text { Produc. } \\
\text { secundaria }\end{array}$ & \begin{tabular}{|c|}
$\begin{array}{c}\text { Comercia- } \\
\text { lización }\end{array}$ \\
\end{tabular} & \begin{tabular}{|c|} 
Adminis- \\
tración
\end{tabular} & \begin{tabular}{|c|}
$\begin{array}{c}\text { Subutil. } \\
\text { planta }\end{array}$ \\
\end{tabular} & Inventario & \begin{tabular}{|l|}
$\begin{array}{c}\text { Faltante de } \\
\text { inventario }\end{array}$ \\
\end{tabular} & Utilidad \\
\hline 1 & 118.8 & 0.0 & 0.0 & 38.0 & 4.5 & 9.3 & 26.2 & $\begin{array}{l}0.0 \\
\end{array}$ & 40.8 \\
\hline 2 & 142.6 & 0.0 & 0.0 & 45.6 & 4.5 & 13.9 & 7.2 & 0.0 & 71.4 \\
\hline 3 & 171.1 & 11.5 & 27.4 & 54.7 & 4.5 & 9.2 & 0.0 & 0.0 & 63.7 \\
\hline 4 & 205.3 & 20.2 & 47.9 & 65.7 & 4.5 & 6.8 & 0.0 & 0.0 & 60.3 \\
\hline 5 & 246.3 & 28.1 & 66.8 & 78.8 & 4.5 & 5.0 & 5.3 & 0.0 & 57.8 \\
\hline 6 & 295.6 & 25.1 & 60.0 & 94.6 & 4.5 & 12.1 & 0.0 & 0.0 & 99.7 \\
\hline 7 & 354.7 & 11.1 & 26.5 & 113.2 & 4.5 & 28.1 & 0.0 & 64.3 & 106.7 \\
\hline Ajuste & -241.2 & 0.0 & 0.0 & -77.2 & 0.0 & 0.0 & 0.0 & 0.0 & -164.0 \\
\hline Total & 1293.2 & 96.0 & 228.2 & 413.8 & 31.5 & 84.3 & 38.6 & 64.3 & 336.4 \\
\hline & $100.0 \%$ & $7.4 \%$ & $17.6 \%$ & $32.0 \%$ & $2.4 \%$ & $6.5 \%$ & $3.0 \%$ & $5.0 \%$ & $26.0 \%$ \\
\hline
\end{tabular}

El cálculo de los porcentajes está en función del ingreso por venta, el cual es de un mil 293 millones 206 mil 100 pesos. Como puede apreciarse, el costo más alto es el de comercialización, el cual representa el $32.0 \%$ del total e incluye distribución, venta y mercadotecnia. Le sigue en orden de importancia el costo de producción secundaria que es $17.6 \%$, el cual incluye producción, mano de obra, envase y empaque. En tercer lugar está el costo de producción primaria, el cual es de 7.4\%, mismo que representa los costos de materia prima (agave), cosecha (jima) y traslado. El costo de subutilización de la planta es $6.5 \%$, el cual es considerablemente alto ya que está muy cercano al costo de producción primaria; mientras que los costos de inventario y faltante de inventario en conjunto representan el $8.0 \%$; en tanto el costo de administración es de sólo el 2.4\%. 
La utilidad total, resultado del modelo, es de 336 millones 400 mil 821 pesos, la cual representa el 26.0\%; pero, si se toma en cuenta que el agave disponible no utilizado representa un costo para los productores y para la cadena de suministro en su conjunto, de 235 millones 363 mil 500 pesos (tomando en cuenta sólo el costo de las plantas, sin jima, ni traslado), entonces la utilidad se reduce a 101 millones 37 mil 321 pesos, la cual representa el $7.8 \%$ del ingreso por venta.

Información obtenida a partir de la corrida del modelo. Escenario alterno

Para este escenario se consideró que el mezcal almacenado (2.1 millones de litros) podría venderse en el corto plazo como una oferta aplicando un descuento del 50\% con el fin de aprovechar al máximo, tanto el agave disponible como la capacidad de producción de las fábricas (en el escenario base los dos primeros años no se produce mezcal, ni se consume agave).

Durante los primeros cinco años el consumo de agave es parcial, pero creciente (gráfica 4); para el sexto y séptimo año se consume el total disponible. A lo largo del horizonte de planeación, el consumo es de sólo el 33\% del total disponible.

\section{Gráfico 4}

Utilización de la capacidad de producción primaria Escenario alterno

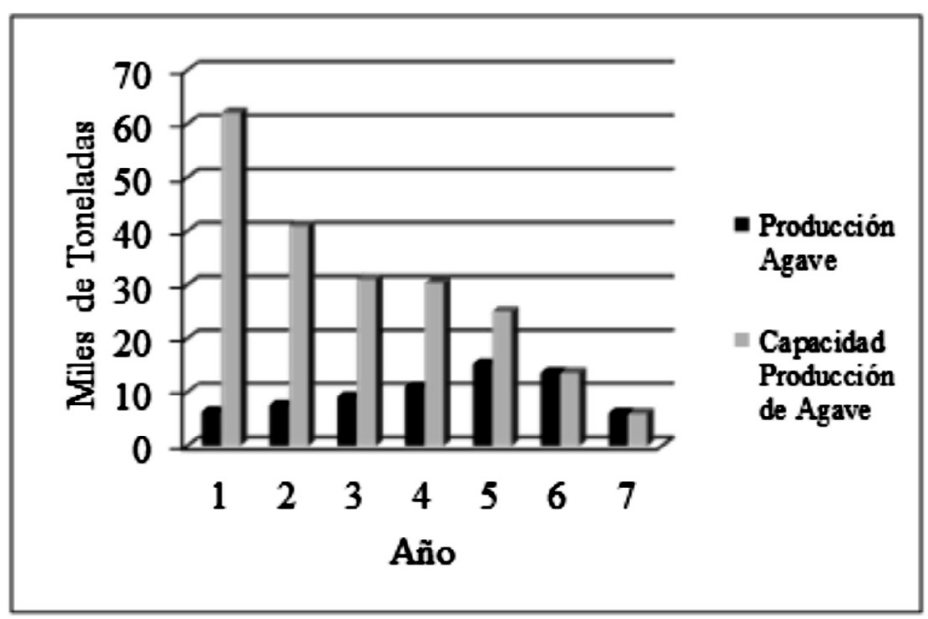


En los cinco primeros años la utilización de la capacidad de producción de mezcal tiene una tendencia creciente que sigue a la tendencia también creciente de la capacidad disponible (gráfica 5). No obstante, decrece en los años sexto y séptimo por la falta de materia prima (agave). Esto también ocasiona que se tenga un faltante de un millón 608 mil 27 litros para cubrir la demanda. A lo largo del horizonte de planeación, la utilización de la capacidad total de las fábricas es de $64 \%$.

\section{Gráfica 5}

Utilización de la capacidad de producción secundaria.

\section{Escenario alterno}

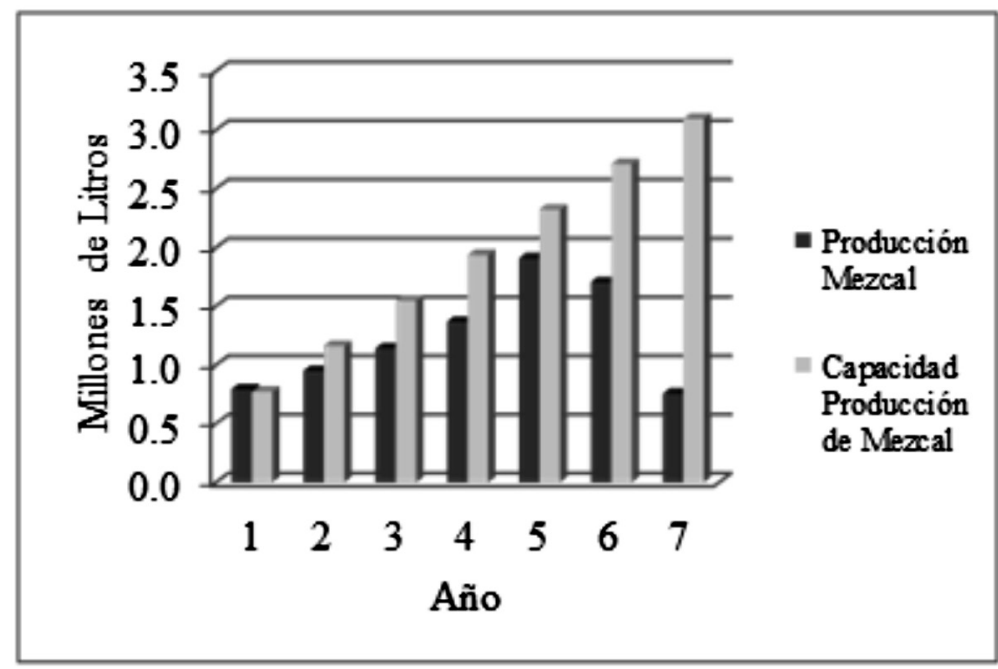

En el cuadro 7 se observan los resultados económicos del modelo para los siete años del escenario alterno. La fila de Ajuste 1 corresponde con el decremento por concepto de venta y comercialización no realizada ya que en el modelo se consideró la opción de penalización por faltante de inventario. La fila de Ajuste 2 corresponde con la venta de los 2.1 millones de litros con un descuento del 50\%; además, no se considera costo de comercialización por esta venta. 


\section{Cuadro 7}

\section{Estructura de costos, venta y utilidad \\ Escenario alterno \\ (Millones de pesos)}

\begin{tabular}{cc|c|c|c|c|c|c|cr}
\hline Año & Venta & $\begin{array}{c}\text { Produc. } \\
\text { Primaria }\end{array}$ & $\begin{array}{c}\text { Produc. } \\
\text { Secundaria }\end{array}$ & $\begin{array}{c}\text { Comercia- } \\
\text { lización }\end{array}$ & $\begin{array}{c}\text { Adminis- } \\
\text { tración }\end{array}$ & $\begin{array}{c}\text { Subutil. } \\
\text { Planta }\end{array}$ & Inventario & $\begin{array}{c}\text { Faltante de } \\
\text { Inventario }\end{array}$ & Utilidad \\
\hline 1 & 118.8 & 11.7 & 27.7 & 38.0 & 4.5 & -0.2 & 0.0 & 0.0 & 37.1 \\
\hline 2 & 142.5 & 14.0 & 33.3 & 45.6 & 4.5 & 2.5 & 0.0 & 0.0 & 42.7 \\
\hline 3 & 171.1 & 16.8 & 40.0 & 54.7 & 4.5 & 4.9 & 0.0 & 0.0 & 50.2 \\
\hline 4 & 205.3 & 20.1 & 47.9 & 65.7 & 4.5 & 6.8 & 0.0 & 0.0 & 60.3 \\
\hline 5 & 246.3 & 28.1 & 66.8 & 78.8 & 4.5 & 5.0 & 5.3 & 0.0 & 57.8 \\
\hline 6 & 295.6 & 25.1 & 59.7 & 94.6 & 4.5 & 12.1 & 0.0 & 0.0 & 99.7 \\
\hline 7 & 354.7 & 11.1 & 26.5 & 113.5 & 4.5 & 28.1 & 0.0 & 64.3 & 106.7 \\
\hline Ajuste 1 & -241.2 & 0.0 & 0.0 & -77.2 & 0.0 & 0.0 & 0.0 & 0.0 & -164.0 \\
\hline Ajuste 2 & 157.5 & 0.0 & 0.0 & 0.0 & 0.0 & 0.0 & 0.0 & 0.0 & 157.5 \\
\hline Total & $\mathbf{1 4 5 0 . 7}$ & $\mathbf{1 2 6 . 9}$ & $\mathbf{3 0 1 . 7}$ & $\mathbf{4 1 3 . 8}$ & $\mathbf{3 1 . 5}$ & $\mathbf{5 9 . 1}$ & $\mathbf{5 . 3}$ & $\mathbf{6 4 . 3}$ & $\mathbf{4 4 8 . 0}$ \\
\hline & $\mathbf{1 0 0 . 0 \%}$ & $\mathbf{8 . 7 \%}$ & $\mathbf{2 0 . 8 \%}$ & $\mathbf{2 8 . 5 \%}$ & $\mathbf{2 . 2 \%}$ & $\mathbf{4 . 1 \%}$ & $\mathbf{0 . 4 \%}$ & $\mathbf{4 . 4 \%}$ & $\mathbf{3 0 . 9 \%}$ \\
\hline
\end{tabular}

En este escenario, el ingreso por venta es de un mil 450 millones 706 mil 100 pesos. Como puede apreciarse, el costo más alto es el de comercialización, el cual representa el $28.5 \%$ del total e incluye distribución, venta y mercadotecnia. Le sigue en orden de importancia el costo de producción secundaria que es $20.8 \%$, el cual incluye producción, mano de obra, envase y empaque. En tercer lugar está el costo de producción primaria, el cual es de $8.7 \%$, mismo que representa los costos de materia prima (agave), cosecha (jima) y traslado. El costo de subutilización de la planta es $4.1 \%$, el cual es menor que el $50 \%$ del costo de producción primaria. Los costos de inventario y faltante de inventario en conjunto representan el $4.8 \%$. El costo de administración es de sólo el 2.2\%.

La utilidad total, resultado del modelo, es de 448 millones 821 mil pesos, la cual representa el $30.9 \%$. Pero si se toma en cuenta que el agave disponible no utilizado representa un costo para los productores y para la cadena de suministro en su conjunto, de 207 millones 119 mil 880 pesos (tomando en cuenta solo el costo de las plantas, sin jima, ni traslado), entonces la utilidad se reduce a 240 millones 880 mil 941 pesos, la cual representa el $16.6 \%$ del ingreso por venta.

\section{Comparación de ambos escenarios}

En el cuadro 8 se presenta una tabla comparativa de los resultados de ambos escenarios: 


\section{Cuadro 8 \\ Comparación ambos escenarios}

\begin{tabular}{l|l|l}
\hline \multicolumn{1}{c|}{ Concepto } & \multicolumn{1}{|c}{ Escenario base } & Escenario alterno \\
\hline Ingreso por Venta & 1293206100 & 1450706100 \\
\hline Costo Total & 956805279 & 1002705279 \\
\hline Utilidad con base al Modelo (\$) & 336400821 & 448000821 \\
\hline Utilidad con base al Modelo (\%) & $26.0 \%$ & $30.9 \%$ \\
\hline Utilidad.- Incluye Desperdicio de Agave (\$) & 101037321 & 240880941 \\
\hline Utilidad.- Incluye Desperdicio de Agave (\%) & $7.8 \%$ & $16.6 \%$ \\
\hline Consumo de Agave Disponible & $25.0 \%$ & $33.0 \%$ \\
\hline Utilización de Capacidad en Fábricas & $48.0 \%$ & $64.0 \%$ \\
\hline
\end{tabular}

El escenario alterno es mejor por las siguientes razones básicas: a) la utilidad del plan total es superior en un $138.4 \%$; b) se consume más agave del disponible, lo cual contribuye a la economía de los productores agrícolas; c) se utiliza un porcentaje mayor de la capacidad instalada de las fábricas, lo cual incentiva a la agroindustria en su conjunto.

\section{Conclusiones}

Aunque algunos de los resultados obtenidos eran "esperados" desde un punto de vista empírico, el presente estudio puede servir de soporte para los tomadores de decisiones en la planeación de los proyectos que requiere esta industria. Por lo que se puede concluir que desde el punto de vista del concepto de cadena de suministro, no existe un enfoque holístico. Según los productores, fabricantes y líderes de opinión hay escasa integración de los procesos productivos de la producción primaria y secundaria, lo cual genera significativos cuellos de botella a lo largo de la misma; por ejemplo, las actividades relacionadas con las áreas de logística de salida y comercial, tanto para la distribución primaria como secundaria, tienen un enorme potencial de mejora.

Las plantaciones de agave están siendo desaprovechadas debido a la reducida demanda de materia prima para la elaboración de mezcal. Esto origina una falta de incentivos para la resiembra. Las corridas del modelo muestran un consumo de agave que va del $25 \%$ al $33 \%$ del total disponible.

La infraestructura de las fábricas para la elaboración de mezcal está siendo subutilizada; actualmente, se emplea menos del 15\% de ésta. Las corridas del 
modelo muestran una potencial utilización de la capacidad en fábricas que va del $48 \%$ al $64 \%$.

\section{Recomendaciones}

Como lo evidenciaron las corridas del modelo táctico, es deseable realizar la venta del mezcal almacenado a través de una estrategia que permita su desplazamiento en el corto plazo ya que de esta manera se reactiva lo antes posible, tanto el uso de plantaciones de agave como de la capacidad instalada en las fábricas.

Debido a que existe una necesidad urgente de contar con capital de trabajo para la cosecha de plantaciones, adquisición de materiales diversos para las fábricas y recursos económicos para los procesos de mercadotecnia, comercialización y distribución, la venta ágil del mezcal almacenado puede generar la liquidez requerida.

Es imprescindible desarrollar un plan mercadológico que le dé al mezcal una revalorización ya que cuenta con ventajas competitivas para lograr así su reconocimiento como una bebida que esté al nivel de las demás bebidas espiritosas del mundo.

Se requiere identificar e implementar el esquema más conveniente de intermediación comercial (un eslabón más en la cadena de suministro) para poner el mezcal a disposición de los mercados meta, valiéndose de las economías de transacción, experiencia, especialización y escala de operaciones de los intermediarios idóneos.

Dado que la comercialización y distribución es la restricción más grande de esta cadena de suministro, urge el desarrollo de un plan comercial que genere una demanda acorde al potencial de esta industria, tanto en el mercado nacional como en el internacional.

El grado de desarrollo que hasta ahora ha alcanzado la industria del mezcal en el estado de Zacatecas puede ser considerado como sobresaliente dados sus logros en diversos campos, como son: infraestructura, identificación geográfica, participación gubernamental, normatividad, certificación e impulso a las clases sociales más necesitadas, entre otros.

La perspectiva de implementar un proceso coordinado y colaborativo entre las micro, pequeñas y mediadas empresas del sector con el fin de fortalecer la cadena 
de suministro es una meta de gran alcance. Dicho objetivo tiene, como premisa fundamental, redoblar los esfuerzos de participación proactiva y colaborativa de los empresarios, lo cual es un requisito indispensable de toda cadena de suministro. Sólo de esta manera se puede lograr sinergia al trabajar en conjunto con la visión de generar más y mejores negocios productivos a partir de emprendedores independientes.

\section{Referencias}

Canizales, M. Romo (2008). De las mieles al mezcal, haciendas y ranchos mezcaleros en Pinos, Zacatecas (1890-1930). México: El Colegio de San Luis.

COMERCAM (2010). Consejo Mexicano de Regulación de la Calidad el Mezcal. Disponible en: www.comercam.org.

Consejo Mexicano de Productores de Maguey Mezcal (2009). Mezcal, nuestra esencia. México: Ámbar Diseño.

Cronbach, Lee J. (1951). Coefficient alpha and the internal structure of tests. Psychometrika 16 (3): 297-334.

David, Fred R. (2008). Strategic Management. 6a. ed. EE.UU.: Macmillan.

Diario Oficial de la Federación (1992). Denominación de Origen Mezcal (DO). México.

Instituto Mexicano de la Propiedad Intelectual (2007). Disponible en:

http://www.impi.gob.mx/impi/jsp/indice_all.jsp. Fecha de consulta: agosto de 2004.

López-Nava, G., J. L. Martínez, J. Cavazos y Y. Mayett (2011). Key elements to design: agave-mezcal supply chain in Mexico. Procedings of the $61^{\text {st }}$ Annual IIE Conference, Reno, Nevada. EE.UU.

Norma Oficial Mexicana NOM-006-SCFI-2005, Bebidas Alcohólicas-Tequila. México. 
Norma Oficial Mexicana NOM-070-SCFI-1994, Bebidas Alcohólicas-Mezcal. México.

Ostle, Bernard (1965). Estadística aplicada. México: Limusa.

Pérez, César (2005). Técnicas estadísticas con SPSS. Madrid: Pearson Prentice Hall.

Porter, Michael (1985). Competitive advantage, creating and sustaining superior performance. EE.UU.: Free Press

Presupuesto Egresos de la Federación 2010 (2009). México.

Presupuesto de la Federación del Año Fiscal 2010 (2009). México.

Smith, Lindsay I. (2002). A tutorial on principal component analysis. Working paper.

Vollman, Thomas E., William L. Berry and D. Clay Whybark (1992). Manufacturing planning and control systems. 3a. ed. EE.UU.: Business One Irwing. 